\title{
High performance pipeline for the calculation of polygenic risk scores
}

\author{
Arina Nostaeva \\ Laboratory of Recombination and \\ Segregation Analysis \\ Institute of Cytology and Genetics \\ SB RAS \\ Novosibirsk, Russia \\ avnostaeva@gmail.com \\ Tatiana Shashkova \\ Laboratory of Recombination and \\ Segregation Analysis \\ Institute of Cytology and Genetics \\ Novosibirsk, Russia
}

\author{
Sodbo Sharapov \\ Laboratory of Recombination and \\ Segregation Analysis \\ Institute of Cytology and Genetics \\ SB RAS \\ Novosibirsk, Russia \\ Yakov Tsepilov \\ Laboratory of Recombination and \\ Segregation Analysis \\ Institute of Cytology and Genetics \\ Novosibirsk, Russia
}

\author{
Yurii Aulchenko \\ Laboratory of Recombination and \\ Segregation Analysis \\ Institute of Cytology and Genetics \\ SB RAS \\ Novosibirsk, Russia \\ PolyKnomics \\ 's-Hertogenbosch, The Netherlands \\ Lennart C. Karssen \\ PolyKnomics \\ 's-Hertogenbosch, The Netherlands
}

\begin{abstract}
A polygenic risk score (PRS) is a value that reflects a person's predisposition to a disease or any other trait which can (partly) be explained by genetic inheritance. PRSs are often used in reports provided by genetic testing companies like 23andMe, Genotek, etc. Another way of using PRSs is to look at the distribution of PRS values for a group of people and compare them, for example, in a case-control study to find casedependent traits. PRS models are usually based on summary statistics data from genome-wide association studies (GWAS) and take into account the linkage disequilibrium (LD) structure. We have created a pipeline for high performance PRS calculations across many traits present in the GWAS-MAP platform. The pipeline only requires individual-level data and provides the ability to select a list of traits. This pipeline will be helpful for scientific groups, working with large amounts of individual genotype data, as well as for individuals with their own personal genotype data.
\end{abstract}

Keywords - genome-wide association study, summary statistics, GWAS, polygenic risk score, linkage disequilibrium, singlenucleotide polymorphisms, GWAS-MAP, database

\section{Introduction}

In the standard approach, a PRS is a linear combination of simple linear regression effect size estimates and allele counts at single-nucleotide polymorphisms (SNPs) that are selected via marker pruning coupled with a $p$-value threshold. Thus, a PRS is the attempt to obtain a single value - the score - for each person and evaluate this person's location - the risk - in the distribution of these values from a sample for a given polygenic trait. For example, for a given disease a PRS shows, for a given person, how large the risk of the disease is relation to other people [1]. Analysis of PRSs requires both genotype data of target individuals as well as GWAS results. GWAS data is needed to obtain effects sizes for further PRS calculation. GWAS give the marginal effect size for each SNP estimated by simple regression models and does not take into account the LD structure. Therefore, in order to construct a PRS model SNP effects should be re-estimated based on the genetic architecture. In case GWAS data is not available in the correct format or there are problems with evaluating the LD structure, the calculation PRS becomes a difficult procedure in terms of resources and time. We created a user-friendly pipeline on top of the GWAS-MAP platform [2] that combines all necessary pre-processing stages with the final
PRS calculation and the ability of using data already available in GWASMAP. It contains basic steps such as the synchronization of individual-level data with summary statistics, the calculation of re-weighted effect estimates from the LD structure and the generation of the PRS. Our high performance pipeline also builds the distribution of polygenic risk scores for a sample of interest and conducts statistical analyses in the case of several target data groups.

\section{Materials and methods}

The GWAS-MAP platform provides access to summary statistics data of more than 7000 traits from different domains including complex traits, metabolomics, proteomics and lipidomics. The complete list of traits can be found through its web-interface called PheLiGe (https://phelige.com/). For reweighting the effect size we chose the SBayesR method [3]. This program uses a shrunk LD matrix and Markov Chain Monte Carlo approach for the estimation of the effects sizes. Two LD matrices are available in our pipeline, one obtained from individual data from the $1000 \mathrm{G}$ project European populations [4] and one from UK biobank data provided by the SbayesR tool. In addition to the standard approach for calculating PRS, this pipeline also allows other methods to be used such as the average approach in which the PRS of individual $i$ is defined as:

$$
P R S_{i}=\frac{\sum_{j} \beta_{j} G_{i j}}{N}
$$

with $\beta_{j}$ the effect size of the $j^{\text {th }}$ SNP, $G_{i j}$ the genotype of the $j^{\text {th }}$ SNP for the $i^{\text {th }}$ individual, and $N$ the number of SNPs included in the PRS of the $i^{\text {th }}$ individual; or the standardization approach:

$$
P R S_{i}=\frac{\frac{1}{N} \sum_{j} \beta_{j} G_{i j}-\operatorname{Mean}(P R S)}{\operatorname{SD}(P R S)}
$$

where SD indicates the standard deviation. Thus, a user is given the choice of model used for computing the PRS.

\section{Results}

Our PRS calculation pipeline implemented on top of the GWAS-MAP platform provides a quick and clear assessment 
of target data without the need for additional processing steps. The program not only provides the user with the necessary data, but also makes it possible to easily use the large number of traits with which the GWAS-MAP platform is constantly updated. Moreover, the option for selecting different models to calculate the PRS makes analyses more flexible. The ability to conduct basic statistical analysis directly in the pipeline allows the user to immediately get results for new data or models. Thus, our pipeline is a tool that a user can access with a minimal set of data and nevertheless get a complete PRS analysis.

\section{ACKNOWLEDGMENT}

This work was partly funded by PolyKnomics BV.

\section{REFERENCES}

[1] Wray NR, Goddard ME, Visscher PM, "Prediction of individual genetic risk to disease from genome-wide association studies," Genome Res., 17(10):1520-8, October 2007.

[2] Gorev D.D., Shashkova T.I., Pakhomov E., "GWAS-MAP: a platform for storage and analysis of the result of thousands of genome-wide association scans," Bioinformatics of Genome Regulation and Structure/Systems Biology, The Eleventh International Conference, C. 43. 2018.

[3] Luke R. Lloyd-Jones, Jian Zeng, "Improved polygenic prediction by Bayesian multiple regression on summary statistics," Nature Communications, vol. 10, 5086, 2019.

[4] Adam Auton, Gonc, alo R. Abecasis, "A global reference for human genetic variation," Nature, vol. 526, p. 68-74, 2015. 